\title{
Auditory and visual similarity effects in recognition and recall
}

\author{
JANE MARANTZ CONNOR and RONALD G. HOYER \\ State University of New York at Binghamton, Binghamton, New York 13901
}

The role of auditory and visual factors in short-term recall and recognition performance was evaluated. Auditory similarity, but not visual similarity, was found to be predictive of the probability of a correct response and response confidence for both types of tests. The results were interpreted as support for a single-trace model of recall and recognition performance.

Three views of the relationship between recall and recognition are identifiable in the experimental literature. One view is that recall and recognition tests are two measures of a single memory trace which differ only in sensitivity (Bahrick, 1965; Postman, Adams, \& Phillips, 1955) or the number of alternative responses entering into the decision process (Norman \& Wickelgren, 1969). A more commonly accepted opinion is that recognition tests measure the availability or strength of a memory trace while recall tests measure both availability and accessibility or retrievability (Bower, Clark, Lesgold, \& Winzenz, 1969; Kintsch, 1970; Murdock, 1968). A third view is that recognition tests measure the strength of a perceptual trace, a trace which is distinct from the verbal memory trace measured by recall tests (Adams, 1967; Adams \& Bray, 1970). One way of testing the viability of theories assuming one vs. two underlying memory traces is to compare the types of errors made by subjects when given recall or recognition tests. If different memory traces are being tapped by the two types of tests, then it seems likely that different error patterns should be found.

The following experiment was designed to evaluate the relative frequencies of visual and auditory confusion errors in recall and recognition tests of visually presented material. Although the importance of auditory factors in recall performance is well established (Conrad, 1964; Conrad, Baddeley, \& Hull, 1966), both auditory and visual factors appear to affect recognition performance (Chase \& Calfee, 1969; Connor, 1972; Gibson \& Yonas, 1966; Krueger, 1970). Numerous procedural differences between the experiments using recall tests and the experiments using recognition tests, however, make direct comparisons between them difficult. For example, scanning rate has been the dependent variable in the

This research was supported by Grant 7R01 MH25344-01 from the National Institute of Mental Health to the senior author. The authors wish to thank C. J. Scheirer for his helpful comments. Requests for reprints should be sent to Jane $M$. Connor, Department of Psychology, State University of New York at Binghamton, Binghamton, New York 13901. recognition literature, while the probability of a correct response has been the dependent variable in the recall literature. It is not clear that these two variables are measuring the same processes; some recent evidence suggests they are not (Scheirer, 1971). Similarly, retention intervals, amount of study time, and types of intervening events have also differed in recognition and recall studies of auditory and visual similarity. Lastly, no study, to our knowledge, has examined the effects of independent variation of auditory and visual similarity on recall and recognition using visual presentation and written tests.

In the following experiment, (a) effects of auditory and visual similarity on recall and recognition were simultaneously evaluated, (b) the same experimental paradigm was used for both types of tests (visual presentation and written responses with confidence ratings), and (c) probability correct and "confidence" were the dependent variables in both cases. If recognition and recall tests are tapping the same underlying memory trace, as many theorists believe, then auditory and visual similarity effects should not depend upon the type of test given. If, on the other hand, visual similarity is a determinant of recognition accuracy under the same circumstances in which auditory confusion errors frequently occur in recall, then Adams' dual trace theory would be supported. A single trace theory could, alternatively, account for this pattern of findings with some additional assumptions. It would be necessary to assume that (1) the memory trace is multidimensional (contains auditory information, visual information, semantic information, etc.), and (2) recognition and recall tests weight the dimensions of the trace differentially. This interpretation is, at this point, indistinguishable from a dual trace theory of recognition and recall.

\section{METHOD}

\footnotetext{
Stimuli

A study slide consisted of 10 letters arranged in two rows. Only one letter was probed on each trial. Probe slides consisted of 10 dashes; each dash corresponded to one of the positions of the $\mathbf{1 0}$
} 
Table 1

Mean Auditory and Visual Similarity Between Target Letter and Distractor for Recognition Tests

\begin{tabular}{lcc}
\hline Distractor Type & $\begin{array}{c}\text { Number of } \\
\text { Listening } \\
\text { Confusions* }\end{array}$ & $\begin{array}{c}\text { Number of } \\
\text { Common Dis- } \\
\text { tinctive Visual } \\
\text { Features** }\end{array}$ \\
\hline High auditory/high visual & 97.9 & 2.1 \\
High auditory/low visual & 61.6 & .3 \\
Low auditory/high visual & 12.1 & 2.3 \\
Low auditory/low visual & 9.4 & .2 \\
\hline *Based on Conrad, 1964 & **Based on Gibson, 1969
\end{tabular}

letters on the study slide. A question mark appeared above one of the dashes, indicating to the recall subjects that they were to write on the answer sheet the letter that had appeared in that position on the study slide. For recognition subjects, a letter appeared above one of the dashes; subjects were to circle the word "yes" on the answer sheet if the probe letter was the letter that had appeared in that position on the study slide. Subjects were to circle the word "no" otherwise.

The letters of the study slides were selected from the set B, C, D, G, P, T, V, Z, F, L, M, N, S, X. Each of these 14 letters was probed eight times, once in each block of 14 trials, yielding a total of 112 trials. The nine filler letters on each slide were randomly chosen without replacement from the remaining 13 letters. Two sets of 112 study slides were constructed. The position of the target letter was randomized in each slide, subject to the constraint that a target letter appearing in a corner position in one set of slides appear in a noncorner position in the other set. Of the 112 recognition tests, each target letter was tested by itself four times and by a distractor four times. Distractors were of either (a) high-auditory/high-visual, $\begin{array}{ll}\text { (b) high-auditory/low-visual, (c) low-auditory/high-visual, or } & \text { (d) }\end{array}$ (d) low-auditory/low-visual similarity to the target letter. Auditory similarity was defined in terms of listening errors, i.e., the frequency with which the test letter was given in response to the auditory presentation of the target letter (Conrad, 1964). Visual similarity was determined by the number of distinctive features the test letter had in common with the target letter (Gibson, 1969, p. 88). The average auditory and visual similarity of the four types of distractors using these measures is shown in Table 1.

\section{Procedure}

Independent groups of subjects received 112 recognition or 112 recall trials. A trial consisted of the presentation of a study slide followed by a probe slide. Each slide was shown for $5 \mathrm{sec}$ by an automatically timed Kodak Carousel projector. Both recall and recognition subjects were asked to rate their confidence in the correctness of each of their responses, from a 1, indicating "no confidence at all," to a 5, indicating "extremely confident." Subjects were instructed to guess if they were not sure of the correct response. Subjects were tested in groups ranging from 10 to 30 in size.

\section{Subjects}

There were 91 subjects in the recall condition and 113 subjects in the recognition condition. The subjects were undergraduates at the State University of New York at Binghamton, participating in the experiment to fulfill a class requirement.

\section{RESULTS}

\section{Recall}

A confusion matrix for recall errors, showing the frequency with which each of the 26 letters of the alphabet was written as a response for each of the 14 stimuli letters, was constructed. The overall error rate for the recall data was $51 \%$, of which $4 \%$ were intrusion errors, that is, responses which did not belong to the target pool of 14 letters. Intrusion errors were excluded from the remainder of the analyses. For each of the 14 target letters, the probability that each of the remaining 13 letters would be supplied as a response to that letter was calculated. A mean confidence rating for correct responses to the 182 $(=14 \times 13)$ possible stimulus-response pairs was also calculated. [Subjects' confidence ratings for erroneous responses were significantly lower than their ratings of correct responses, $t(90)=16.92$. $p<.05$.$] A measure of the auditory similarity of each$ of the pairs was obtained from Conrad's (1964) listening data showing the probability that each response letter would be confused with each stimulus letter when the stimulus letter was presented auditorially against a background of white noise. The measure of the visual similarity of each of the pairs that was used was the number of visual distinctive features that each of the letters had in common (Gibson, 1969, p. 88).

Auditory similarity predicted both the probability that a particular letter would be erroneously given in response to a target letter, $\mathrm{r}(180)=.40, \mathrm{p}<.05$, and the confidence of the subjects in their responses. $r(180)=.33, p<.05$. Visual similarity was not predictive of either confusion errors, $r(180)=.11$, $p>.05$, or confidence, $r(180)=-.01, p>.05$. These results are quite similar to those obtained by Conrad (1964), which imply that auditory similarity and not visual similarity predicts errors in written recall of visually presented material.

\section{Recognition}

For each subject, the probability of a correct "no" response to a distractor was calculated as a function of the four distractor types. Mean confidence ratings for correct rejections of distractors, which were higher than ratings of incorrect acceptances, $F(1,112)=$ 21.8, $p<.05$, mean square error $(\mathrm{MSE})=.610$, were also calculated. Analyses of variance showed a reliable difference between high- and low-auditory distractors in both probability correct, $F(1,112)=17.07$, $\mathrm{p}<.05, \mathrm{MSE}=.098$, and confidence level, $\mathrm{F}(1,112)$ $=10.60, \mathrm{p}<.05, \mathrm{MSE}=.955$ (see Table 2 ). A small, but significant, effect of visual similarity was found for the two measures, $F(1,112)=5.05$, $\mathrm{p}<.05, \mathrm{MSE}=.096$, and $\mathrm{F}(1,112)=6.41, \mathrm{p}<.05$, MSE $=1.209$. The interaction between auditory and visual similarity was not significant for probability correct. $\mathrm{F}(1,112)=2.21, \mathrm{p}>.05, \mathrm{MSE}=.112$, or for confidence ratings, $F(1,112)=2.18, \mathrm{p}>.05$, MSE $=1.164$.

Further analysis suggests that the auditory similarity between target letters and distractors, not 
Table 2

Recognition Data: Mean Probability Correct and Confidence Rating for Each Distractor Type

\begin{tabular}{lcc}
\hline Distractor Type & $\begin{array}{c}\text { Probability } \\
\text { Correct }\end{array}$ & $\begin{array}{c}\text { Confidence } \\
\text { Rating* }\end{array}$ \\
\hline High auditory/high visual & .853 & 3.95 \\
High auditory/low visual & .858 & 4.06 \\
Low auditory/high visual & .873 & 4.07 \\
Low auditory/low visual & .903 & 4.10 \\
\hline
\end{tabular}

*Confidence ratings ranged from 1 to 5 in increasing order of confidence.

the visual similarity, is the determinant of recognition performance. Whereas probability correct and confidence rating for each stimulus-probe pair was correlated with listening errors, $r(54)=-.41$, $\mathrm{p}<.01$, and $\mathrm{r}(54)=-.31, \mathrm{p}<.05$, respectively, these variables did not correlate with visual similarity, $r(54)=-.05, p>.05$, and $r(54)=-.01, p>.05$. The main effect of visual similarity that was found was probably due to the fact that visually similar pairs were also more auditorially similar (see Table 1). The pairs in the high-auditory condition were, on the other hand. well matched for visual similarity. This conclusion is supported by the results of two analyses of covariance. When auditory similarity was used as a covariate, no effect of visual similarity was obtained, $\mathrm{F}<1$. When visual similarity was used as a covariate, the effect of auditory similarity was reduced, but still present, $F(1.111)=3.9, \mathrm{p}<.05$.

\section{DISCUSSION}

The results of this experiment, using visual presentation and written tests, suggest that auditory, not visual, similarity is a determinant of recall and recognition accuracy. One might question, however, whether the scales of auditory and visual similarity were appropriately sensitive to variations in the two dimensions. Although we would certainly not wish to claim that the two scales are equally sensitive to such variations, the results of other studies using the same scales indicate that each of the scales is sufficiently sensitive to produce performance changes in other situations (Connor, 1972; Gibson \& Yonas, 1966). This concern is further diminished by our finding that visual similarity, when auditory similarity was controlled, had virtually no effect. If we had found that both types of similarity had an effect differing in amount, then the question of scale sensitivity would be a serious problem. Given the present pattern of results and the prior literature, we believe the conclusion that auditory similarity is a determinant of both result and recognition accuracy is warranted.

This conclusion is consistent with the view that recall and recognition tests are based upon the same underlying memory trace (an auditory-based code in the present experiment), and that differences between them are based upon other factors, e.g., retrieval. Although the present data are relatively unambiguous in this respect, it would be a mistake to conclude that storage differences are never implicated in recall-recognition differences. Frost (1972), for example, found that visual similarity had different effects on memory for pictorial stimuli when subjects were expecting a recall test or a recognition test. The results of Tversky (1973) also suggest that different types of information may be used to pass a recall test or a recognition test. The type of stimuli used in this experiment and the temporal requirements may have limited the subjects' ability to encode and store the stimuli differently for recall and recognition tests. That is, with only $5 \mathrm{sec}$ available to study 10 letters, subjects in both groups may have been forced to emphasize the auditory-verbal aspects of the stimuli to the exclusion of the visual characteristics. With a smaller memory load and a shorter retention interval, it might be possible to demonstrate the use of different memory traces or encoding processes for recognition and recall tests of letter stimuli.

However, the use of shorter delays and smaller memory loads presents other problems. Under such conditions, recognition performance is virtually error-free and probability correct is no longer a meaningful dependent variable. The use of reaction time as a dependent variable for recognition tests is both useful and informative, but presents difficulties with recall tests. Thus parametric studies of the factors affecting recall and recognition are difficult to design. Until such studies are completed, the assessment of the merits of the various theories will be incomplete. Although the evidence from reaction time studies suggests that visual characteristics probably do play a significant role in recognition performance (Chase \& Calfee, 1969; Connor, 1972; Posner et al., 1969), it is difficult to compare this effect to recall performance because of large procedural variations.

\section{REFERENCES}

Adams. J. A. Human memory. New York: McGraw-Hill. 1967.

ADAMs, J. A.. \& BRAY. N. W. A closed-loop theory of pairedassociate verbal learning. Psychological Review, 1970, 77. $385-504$.

Barrick, H. P. The ebb of retention. Psychological Review, 1965, 72, 60-73.

Bower, G. H., Clark, M. C., Lesgold, A. M., \& Winzenz, D. Hierarchical retrieval schemes on recall of categorized word lists. Journal of Verbal Learning and Verbal Behavior, 1969, 8 , 323-343.

Chase, W. G., \& Calfee, R. C. Modality and similarity effects in short-term recognition memory. Joumal of Experimental Psychology, 1969, 81, 510-514.

Connor, J. M. Serial and parallel encoding processes in memory and visual search. Joumal of Experimental Psychology. 1972, 96. 363-370. 
Conrad, R. Acoustic confusions in immediate memory. British Journal of Psychology, 1964, 55, 75-84.

Conrad, R., Baddeley, A. D., \& Hull, A. J. Rate of presentation and the acoustic similarity effect in short-term memory. Psychonomic Science, 1966, 5, 233-234.

Frost, N. Encoding and retrieval in visual memory tasks. Journal of Experimental Psychology, 1972, 95, 317-326.

GIBson, E. Principles of perceptual leaming and development. New York: Appleton-Century-Crofts, 1969.

Grbson, E. J., \& Yonas, A. A developmental study of the effects of visual and auditory interference on a visual scanning task. Psychonomic Science, 1966, 5, 163-164.

KINTsCH, W. Models for free recall and recognition. In D. A. Norman (Ed.), Models of human memory. New York: Academic Press, 1970.

KrUEGer, L. E. The effect of acoustic confusability on visual search. American Joumal of Psychology, 1970, 83, 389-400.

MurDocz, B. B., JR. Modality effects in short-term memory: Storage or retrieval? Joumal of Experimental Psychology, $1968,77,79.86$.
Norman, D. A., \& Wickelgren, W. A. Strength theory of decision rules and latency in short-term memory. Journal of Mathematical Psychology, 1969, 6. 192-208.

Posner, M. I., Boies, S. J., Eichelman, W. H., \& Taylor, R. L. Retention of visual and name codes of single letters. Journal of Experimental Psychology Monographs, 1969, 79, $1-13$.

Postman, L., Adams, P. A., \& Phillips, L. W. Studies in incidental learning: II. The effects of association value and of the method of testing. Joumal of Experimental Psychology, 1955, 49, 1-10.

Screirer, C. J. Effect of cueing, modality, and effective contiguous time on response latency in short-term memory. Journal of Experimental Psychology, 1971, 88, 429-432.

TVERSKY, B. Encoding processes in recognition and recall. Cognitive Psychology, 1973, 5, 275-287.

(Received for publication June 15, 1975; revision accepted September 2,1975 .) 